\section{Entrenamiento físico en personas con enfermedad renal crónica avanzada: beneficios de su implementación en la práctica clínica}

\author{
HANS MÜLLER-ORTIZ ${ }^{2,3,5,6,7}$, CRISTIAN PEDREROS-ROSALES ${ }^{1,2,3,7}$, \\ ALDO VERA-CALZARETTA ${ }^{2,4, \mathrm{a}, \mathrm{c}}$, ALEXIS GONZÁLEZ-BURBOA ${ }^{2,4, \mathrm{~b}, \mathrm{~d}}$, \\ CARLOS ZÚÑIGA-SAN MARTÍN ${ }^{2,3,5,6,7}$, \\ MARÍA SOLEDAD OLIVEROS-ROMERO ${ }^{2,3}$
}

\section{Exercise training in advanced chronic kidney disease}

Chronic kidney disease is associated with an increase in cardiovascular risk and mortality, and a decrease in the health-related quality of life. It is known that the physical capacity of patients with chronic kidney disease is lower than their healthy counterparts. It also decreases progressively after the start of hemodialysis, even leading to the loss of functional independence. There is a positive association between exercise and improvement of many targets in chronic kidney disease, especially in dialysis patients, such as cellular apoptosis, immune improvement and inflammation. In addition, several clinical studies and systematic reviews show an improvement in different clinical and laboratory variables and suggest a lower mortality, proving a reduction of health care economic costs. Exercise intervention in dialysis is safe. Although different guidelines promote exercise, it is necessary to work on its implementation on a large scale.

(Rev Med Chile 2019; 147: 1443-1448)

Key words: Exercise; Quality of Life; Renal Dialysis; Renal Insufficiency

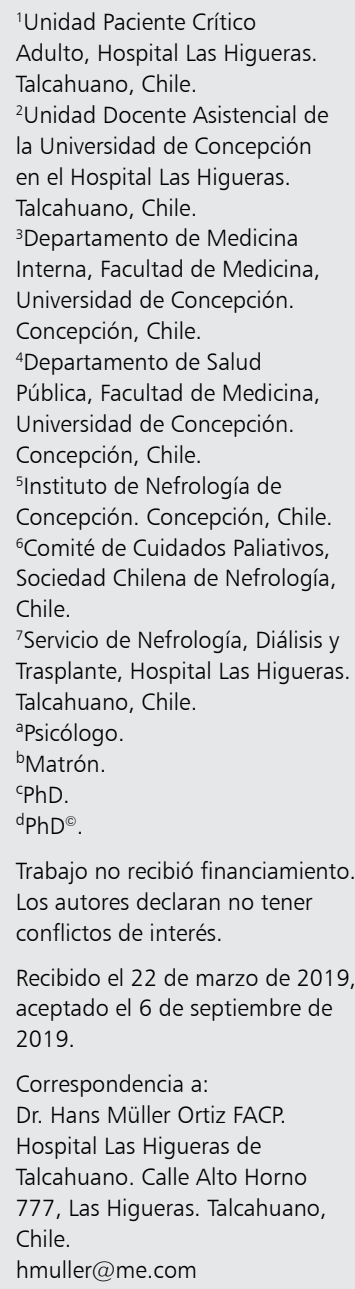

E ste trabajo tiene por propósito contribuir a la discusión sobre la importancia de la incorporación del entrenamiento físico como parte del tratamiento médico integral de los pacientes con enfermedad renal crónica (ERC) avanzada. Para ello, en primer lugar, se aborda la situación actual de esta enfermedad. En segundo lugar, se analiza la capacidad física del paciente con ERC. Luego, se describe la evidencia relativa a los beneficios del entrenamiento físico en los mismos $y$, finalmente, se señalan algunas consideraciones.

\section{Situación de la ERC avanzada}

En el mundo, la ERC ha tenido un incremento acelerado en las últimas décadas, constituyendo un problema de salud pública ${ }^{1,2}$.

El tratamiento de la ERC avanzada (etapa 5) y el acceso universal a las terapias de sustitución renal -diálisis y trasplante- han tenido avances significativos desde sus inicios en la década de 1960-69 al presente ${ }^{3,4}$.

En este escenario, en Chile, la tasa de pacientes en hemodiálisis ha tenido un incremento significativo, variando de 12,7 pacientes por millón de personas en 1980 a 1.208 por millón de personas en $2017^{5}$.

Si bien, la diálisis mejora la sintomatología y sobrevida, no cura la enfermedad. Además, tiene morbimortalidad asociada-especialmente cardio- 
vascular-y afecta significativamente la calidad de vida de los pacientes ${ }^{6,7}$. Por ejemplo, se describe que en estos pacientes, la sintomatología física y emocional tiene una alta prevalencia, y que el número e intensidad de sus síntomas es comparable al reportado en pacientes con cáncer o $\mathrm{SIDA}^{8}$. Por otra parte, aun siendo una terapia poco frecuente, es de muy alto costo. Como ejemplo, en Chile, solo el gasto de la hemodiálisis crónica (HDC) representa 22\% del presupuesto GES ${ }^{9}$. Lo anteriormente señalado avala la necesidad de crear nuevas estrategias de intervención complementarias a la diálisis.

\section{Capacidad física del paciente en diálisis}

La capacidad física de los pacientes con ERC en etapa 5 equivale solo a $60 \%$ de una población de la misma edad con función renal normal ${ }^{10} \mathrm{y}$ va progresivamente disminuyendo después del inicio de la hemodiálisis crónica (HDC) ${ }^{11}$. Estos bajos niveles de actividad física se asocian a pérdida de masa muscular, conduciendo a debilidad, bajo rendimiento físico y a una limitación de la movilidad, con pérdida de la independencia funcional ${ }^{12,13}$. Por ejemplo, la sarcopenia es más prevalente en sujetos con ERC en etapas 3-5 que en la población normal ${ }^{14} \mathrm{y}$ puede estar presente en un tercio de los pacientes en hemodiálisis ${ }^{15}$.

Dado que caminar "desafía" a los sistemas cardiopulmonar, circulatorio, nervioso y musculoesquelético, la velocidad de la marcha constituye un marcador confiable del estado de salud. Existe evidencia en estudios de cohortes que demuestran una asociación inversa entre la velocidad de marcha y el número de hospitalizaciones, dificultades en las actividades de la vida diaria y mortalidad en pacientes con $\mathrm{HDC}^{16,17}$. Además, se conoce que el sedentarismo, por sí solo, es un factor de riesgo de mortalidad en pacientes en hemo y peritoneodiálisis, incluso tan importante como la hipoalbuminemia ${ }^{18}$.

\section{Beneficios del entrenamiento físico en pacientes con ERC avanzada: ¿Qué nos dice la evidencia?}

Existe importante evidencia experimental y clínica que demuestra que el aumento de la actividad física en estos pacientes contribuye a la mejoría de múltiples variables biopsicosociales, pudiendo, además, impactar positivamente en su sobrevida.

Un interesante trabajo experimental en ratones ${ }^{19}$, demostró que el ejercicio físico puede mejorar la ERC a través de la regulación de las vías intrínseca y extrínseca de la apoptosis, lográndose un mayor beneficio a mayor duración de este, así como también puede mejorar la función inmune y ejercer efectos antiinflamatorios en pacientes con ERC en etapa prediálisis, particularmente si se realiza de manera constante ${ }^{20}$. En humanos, también se ha descrito que en pacientes con ERC etapas 3 y 4 que un aumento en la actividad física podría enlentecer la caída de la tasa de filtración glomerular ${ }^{21,22}$.

Un estudio de cohorte de Manfredini y cols. ${ }^{23}$, que evaluó la respuesta de las células progenitoras endoteliales circulantes (CPEC) en un programa de 6 meses de ejercicio de moderada intensidad en pacientes en hemodiálisis, demostró un aumento significativo y selectivo en el número de estas. Además, señaló como fundamento que el ejercicio físico induce una remodelación vascular marcada al aumentar la angiogénesis y la arteriogénesis, generando un aumento del número de CPEC, las que podrían desempeñar un papel en la progresión de la enfermedad cardiovascular en pacientes con $\mathrm{ERC}$, los cuales generalmente muestran un número reducido de estas células.

El estudio multicéntrico Dialysis Outcomes and Practice Patterns Study (DOPPS) ${ }^{24}$, diseñado para evaluar múltiples variables de la terapia hemodialítica en una cohorte prospectiva de más de 20.000 pacientes en HDC de diversos países, concluyó que el riesgo de morir ajustado por diversas variables (demográficas, condiciones de comorbilidad, uso de catéter, entre otras) se redujo significativamente en los pacientes que practicaban ejercicio al menos una vez por semana $(\mathrm{p} \leq 0,0002)$, disminuyendo aun más al aumentar la frecuencia del mismo ( $\mathrm{p}<0,0001)$. También se reportaron mejores resultados en ámbitos propios de la calidad de vida relacionada con la salud ( $\mathrm{p}<0,0001)$, tales como funcionalidad física, calidad del sueño, dolor corporal, síntomas depresivos y apetito.

En 2011, The Cochrane Collaboration ${ }^{25}$ publicó un metaanálisis de 32 estudios experimentales que evaluaron el efecto del entrenamiento con ejercicio físico regular (de ocho o más semanas de duración) sobre el estado nutricional, inflamación, 
función cardiaca, actividad física, estado físico, funcionalidad y bienestar psicológico de adultos con ERC. Respecto al tipo de intervención implementada, la más frecuente fue el entrenamiento con ejercicio cardiovascular, seguido de entrenamiento mixto de resistencia física y cardiovascular, entrenamiento de resistencia y yoga. Además, se consideraron intervenciones con sesiones de ejercicio supervisadas y no supervisadas. Los resultados mostraron que el entrenamiento con ejercicio físico, sin importar el tipo, la duración de la intervención o la existencia de supervisión durante este, mejoró significativamente la capacidad aeróbica $(\mathrm{p}<0,00001)$, la fortaleza muscular $(\mathrm{p}<0,00001)$, la capacidad de marcha $(\mathrm{p}=0,003)$, la presión arterial diastólica en reposo $(\mathrm{p}=0,009)$, la presión arterial sistólica en reposo $(\mathrm{p}=0,002)$, la frecuencia cardíaca máxima $(\mathrm{p}=0,002)$, la frecuencia cardíaca en reposo $(p=0,002)$ y algunos marcadores nutricionales como la albúmina $(\mathrm{p}=0,02)$, la prealbúmina $(\mathrm{p}=0,002)$ y la ingesta energética $(\mathrm{p}=0,03)$.

Otra revisión sistemática y metaanálisis de 24 ensayos clínicos controlados y con distribución aleatoria, publicado en $2014^{26}$, evaluó el efecto del ejercicio intradialítico sobre algunos indicadores clínicos de pacientes con HDC. Se encontraron mejoras en la eficacia de la diálisis $(\mathrm{Kt} / \mathrm{V})$ $(\mathrm{p}=0,04)$, en el consumo máximo de oxígeno $\left(\mathrm{VO}_{2}\right.$ peak) $(\mathrm{p}<0,001)$ y en el desempeño del test de caminata de 6 min (6-MWD). La mejoría del $\mathrm{Kt} / \mathrm{V}$ también se ha comunicado en otros estudios y se podría explicar por el incremento del flujo sanguíneo a nivel muscular durante el ejercicio, lo que permitiría aumentar la remoción de tóxicos por el dializador ${ }^{27}$.

Otros estudios han reportado potenciales beneficios de la actividad física sobre aspectos neurológicos, disminución del tratamiento antihipertensivo y enfermedad osteometabólica en pacientes en diálisis. Por ejemplo, frente al deterioro cognitivo que desarrollan estos pacientes, cuya prevalencia puede alcanzar hasta $70 \%^{28-30}$, existen opiniones a favor de que la actividad física induciría una mejora en el funcionamiento cognitivo, pudiendo retardar la progresión de este ${ }^{31}$.

También se han comunicado beneficios del ejercicio en el síndrome de piernas inquietas (SPI), cuadro complejo y frecuente en los pacientes en diálisis, que puede afectar a casi a un tercio de ellos. Al respecto, una revisión sistemática publicada en
2015 sintetizó 14 estudios que evaluaron la efectividad de las opciones terapéuticas actuales del SPI urémico implementadas en pacientes dializados crónicos (HDC o peritoneodiálisis). Los resultados mostraron que el ejercicio fue beneficioso, tanto para reducir la puntuación de gravedad de los síntomas (disminución entre $28 \%$ y $58 \%$ ), como para mejorar la calidad de vida (mejoría entre $18 \%$ y $25 \%$ ) y la calidad del sueño (mejoría entre $20 \%$ y $50 \%)^{32}$.

Del mismo modo, Miller y cols. ${ }^{33}$ observaron que $54 \%$ de los pacientes con actividad física intradiálisis redujeron la cantidad de fármacos antihipertensivos a los seis meses versus $12,5 \%$ en el grupo control $(\mathrm{p}=0,008)$, con una disminución de los costos económicos asociados (ahorro USD 885/paciente-año; $p=0,005$ ). Esto, según los autores, puede justificar el capital inicial para la adquisición de equipos y el recurso humano especialista para la implementación de programas de ejercicios.

Otra condición que acompaña a la ERC es el hiperparatiroidismo secundario, trastorno que afecta de forma directa la calidad de vida y aumenta la mortalidad en esta población ${ }^{34}$. Un estudio transversal, cuyo objetivo fue valorar la relación de los niveles de hormona paratiroidea (PTH) con la actividad física en pacientes en HDC, demostró que los niveles de PTH intacta se asociaron inversamente con la actividad física diaria y observó que la asociación entre la PTH intacta y la actividad diaria no fue lineal y esta última fue menor solo en pacientes con niveles más bajos y más altos de PTH intacta ${ }^{35}$.

Otro beneficio del ejercicio, en particular el de resistencia, es su utilidad para mejorar la densidad mineral ósea en pacientes en HDC, describiéndose en un estudio experimental una reducción significativa del porcentaje de osteoporosis en el grupo que realizó ejercicio ${ }^{36}$. También se han comunicado beneficios de programas de ejercicio intradiálisis adaptados para pacientes ancianos con alta comorbilidad en HDC (mayores a 75 años), observando mejoras significativas en la fuerza muscular, capacidad funcional, sintomatología depresiva y calidad de vida relacionada a salud $(\mathrm{CVRS})^{37}$. Este hecho es relevante, considerando que esta población está en continuo crecimiento ${ }^{38}$.

En Chile, se publicó un estudio piloto sobre entrenamiento físico durante hemodiálisis ${ }^{39}$, cuyo objetivo fue evaluar los efectos de un programa 
de entrenamiento de 16 semanas con ejercicios aeróbicos y de resistencia en pacientes sometidos a hemodiálisis crónica. Este logró demostrar en el grupo intervenido mejoras significativas en el test de caminata de seis minutos y en la fuerza del cuádriceps.

En el contexto de estos pacientes la actividad física podría asociarse a efectos adversos, no obstante, en condiciones controladas, estos son bajos $^{26,32,38,40}$. Al respecto, se han descrito lesiones musculares y tendíneas (especialmente en pacientes con hiperparatiroidismo grave), dolores musculares (generalmente transitorios), y episodios aislados de hipoglicemia e hipotensión. Sin embargo, se debe tener presente que en muchos de los estudios controlados no se hacía referencia a los efectos adversos o se excluyeron los pacientes con alteraciones médicas importantes ${ }^{41}$.

En los últimos años se han descrito variados programas de ejercicio físico en pacientes con diálisis, tanto domiciliarios como intradiálisis, pero aún no hay consenso en cuanto al tipo, frecuencia y duración ideal de estos ${ }^{42}$. Frente al desafío de homogeneizar estas variables y proponer guías locales de consenso para su implementación, es importante señalar que profesionales como los kinesiólogos y profesores de educación física, cuyas competencias curriculares incorporan una sólida formación en entrenamiento físico, podrían contribuir a estos programas ${ }^{43}$.

\section{Consideraciones finales}

Pese a que aún es necesario seguir investigando para esclarecer los mecanismos mediante los cuales la actividad física influye positivamente en el estado de salud de las personas con ERC avanzada, la evidencia descrita ofrece una visión general sobre el entrenamiento físico y su impacto favorable sobre aspectos clínicos, de laboratorio, terapéuticos, CVRS y potencial sobrevida de los pacientes con ERC avanzada, sin olvidar el control de algunos costos económicos asociados.

Hace años las guías KDOQI (Kidney Disease Outcomes Quality Initiative ${ }^{44}$ ya señalaban la importancia de la actividad física en los pacientes con ERC, no obstante, los programas de entrenamiento físico formales en centros de diálisis no existen o son escasos ${ }^{45}$. Chile no es la excepción. Así, pese a que nuestro país ha logrado garantizar el acceso universal al tratamiento dialítico, la incorporación de estas intervenciones continúa siendo un desafío pendiente.

Finalmente, sería recomendable incorporar contenidos relacionados con el rol del ejercicio físico en la terapia de los pacientes con ERC en reuniones de divulgación científica y de especialistas, así como en los programas de formación de residentes en nefrología adulto/pediátricos y currículos de otras disciplinas relacionadas con la atención de pacientes renales.

\section{Referencias}

1. Crews DC, Bello AK, Saadi G. 2019 World Kidney Day Editorial - burden, access, and disparities in kidney disease. J Bras Nefrol 2019; 41 (1): 1-9.

2. Luyckx VA, Tonelli M, Stanifer JW. The global burden of kidney disease and the sustainable development goals. Bull World Health Organ 2018; 96 (6): 414-22D.

3. Bestard O, Campistol JM, Morales JM, Sánchez-Fructuoso A, Cabello M, Cabello V, et al. Avances en la inmunosupresión para el trasplante renal: Nuevas estrategias para preservar la función renal y reducir el riesgo cardiovascular. Nefrología 2012; 32 (3): 374-84.

4. López Gómez JM. Avances en hemodiálisis clínica: accesos vasculares y pacientes de edad avanzada. Nefrologia 2008; 28 (Suppl 5): 67-70.

5. Poblete H. XXXVIII Cuenta de Hemodiálisis Crónica en Chile 2018. [Internet]. Chile: Sociedad Chilena de Nefrología; 2018. [ Citado el 22 de marzo de 2019]. Disponible en: https://www.nefro.cl/v2/biblio/registro/24. pdf.

6. Go AS, Chertow GM, Fan D, McCulloch CE, Hsu CY. Chronic kidney disease and the risks of death, cardiovascular events and hospitalization. N Engl J Med 2004; 351 (13): 1296-305.

7. Zúñiga C, Dapueto J, Müller H, Kirsten L, Alid R, Ortiz L. Evaluación de la calidad de vida en pacientes en hemodiálisis crónica mediante el cuestionario "Kidney Disease Quality of Life (KDQOL-36)". Rev Med Chile 2009; 137 (2): 200-7.

8. Zúñiga C. Cuidados de soporte/paliativos en diálisis. ¿Por qué, cuándo y cómo? Dial Traspl 2014; 35 (1): 20 6.

9. Gobierno de Chile. Guía Clínica: Prevención Enfermedad Renal Crónica. [Internet]. Santiago: Ministerio de Salud; 2008. [Citado el 22 de marzo de 2019]. Disponible en: https://www.minsal.cl/portal/url/item/8173e4c747591cede04001011e016819.pdf. 
10. Painter P, Messer-Rehak D, Hanson P, Zimmerman SW, Glass NR. Exercise capacity in hemodialysis, CAPD, and renal transplant patients. Nephron 1986; 42 (1): 47-51.

11. Kurella Tamura M, Covinsky KE, Chertow GM, Yaffe $\mathrm{K}$, Landefeld CS, McCulloch CE. Functional status of elderly adults before and after initiation of dialysis. $\mathrm{N}$ Engl J Med 2009; 361 (16): 1539-47.

12. Morishita S, Tsubaki A, Shirai N. Physical function was related to mortality in patients with chronic kidney disease and dialysis. Hemodialysis International 2017; 21(4): 483-9.

13. Roshanravan B, Gamboa J, Wilund K. Exercise and CKD: Skeletal muscle dysfunction and practical application of exercise to prevent and treat physical impairments in CKD. Am J Kidney Dis. 2017; 69(6): 837-52.

14. Foley R, Wang Ch, Ishani A, Collins A, Murray A. Kidney Function and Sarcopenia in the United States General Population: NHANES III. Am J Nephrol 2007; 27 (3): 279-86.

15. Kim JK, Choi SR, Choi MJ, Kim SG, Lee YK, Noh JW, et al. Prevalence of and factors associated with sarcopenia in elderly patients with end-stage renal disease. Clin Nutr 2013; 33 (1): 64-8.

16. Kunter NG, Zhang R, Huang Y, Painter P. Gait speed and mortality, hospitalization, and functional status change among hemodialysis patients: a US Renal Data System special study. Am J Kidney Dis 2015; 66 (2): 297 304.

17. Shimoda T, Matsuzawa R, Yoneki K, Harada M, Watanabe T, Yoshida A, et al. Combined contribution of reduced functional mobility, muscle weakness, and low serum albumin in prediction of all-cause mortality in hemodialysis patients: a retrospective cohort study. J Ren Nutr 2018; 28 (5): 302-8.

18. O'Hare AM, Tawney K, Bacchetti P, Johansen KL. Decreased survival among sedentary patients undergoing dialysis: results from the dialysis morbidity and mortality study wave 2. Am J Kidney Dis. 2003; 41(2): 447-54.

19. Chen KC, Peng CC, Hsieh CL, Peng RY. Exercise ameliorates renal cell apoptosis in chronic kidney disease by intervening in the intrinsic and the extrinsic apoptotic pathways in a rat model. Evid Based Complement Alternat Med 2013; 2013: 368450.

20. Viana JL, Kosmadakis GC, Watson EL, Bevington A, Feehally J, Bishop NC, et al. Evidence for anti-inflammatory effects of exercise in CKD. J Am Soc Nephrol 2014; 25 (9): 2121-30.

21. Greenwood SA, Koufaki P, Mercer TH, MacLaughlin HL, Rush R, Lindup H, et al. Effect of exercise training on estimated GFR, vascular health, and cardiorespiratory fitness in patients with CKD: a pilot randomized controlled trial. Am J Kidney Dis 2015; 65 (3): 425-34.

22. Castaneda C, Gordon PL, Uhlin KL, Levey AS, Kehayias JJ, Dwyer JT, et al. Resistance training to counteract the catabolism of a low-protein diet in patients with chronic renal insufficiency: a randomized, controlled trial. Ann Intern Med 2001; 135 (11): 965-76.

23. Manfredini F, Rigolin GM, Malagoni AM, Soffritti S, Boari B, Conconi F, et al. Exercise capacity and circulating endothelial progenitor cells in hemodialysis patients. Int J Sports Med 2007; 28 (5): 368-73

24. Tentori F, Elder SJ, Thumma J, Pisoni RL, Bommer J, Fissell RB, et al. Physical exercise among participants in the Dialysis Outcomes and Practice Patterns Study (DOPPS): correlates and associated outcomes. Nephrol Dial Transplant 2010; 25( 9): 3050-62.

25. Heiwe S, Jacobson SH. Exercise training for adults with chronic kidney disease. Cochrane Database Syst Rev 2011; 10: CD003236.

26. Sheng K, Zhang P, Chen L, Cheng J, Wu C, Chen J. Intradialytic exercise in hemodialysis patients: a systematic review and meta-analysis. Am J Nephrol 2014; 40 (5): 478-90.

27. Parsons TL, Toffelmire EB, King-VanVlack CE. Exercise training during hemodialysis improves dialysis efficacy and physical performance. Arch Phys Med Rehabil 2006; 87 (5): 680-87.

28. Murray AM, Tupper DE, Knopman DS, Gilbertson DT, Pederson SL, Li S, et al. Cognitive impairment in hemodialysis patients is common. Neurology 2006; 67 (2): 216-23.

29. Rodríguez-Angarita CE, Sanabria-Arenas RM, Vargas-Jaramillo JD, Ronderos-Botero I. Cognitive impairment and depression in a population of patients with chronic kidney disease in Colombia: a prevalence study. Can J Kidney Health Dis 2016; 3 (1): 26.

30. Yaffe K, Ackerson L, Tamura MK, Le Blanc P, Kusek JW, Sehgal AR, et al. Chronic kidney disease and cognitive function in older adults: findings from the chronic renal insufficiency cohort cognitive study. J Am Geriatr Soc 2010; 58 (2): 338-45.

31. Bronas UG, Puzantian H, Hannan M. Cognitive impairment in chronic kidney disease: vascular milieu and the potential therapeutic role of exercise. Biomed Res Int 2017; 2017: 2726369.

32. Sakkas GK, Giannaki CD, Karatzaferi C, Maridaki M, Koutedakis Y, Hadjigeorgiou GM, et al. Current trends in the management of uremic restless legs syndrome: a systematic review on aspects related to quality of life, cardiovascular mortality and survival. Sleep Med Rev 2015; $21: 39-49$.

33. Miller BW, Cress CL, Johnson ME, Nichols DH, Schnit- 
zler MA. Exercise during hemodialysis decreases the use of antihypertensive medications. Am J Kidney Dis 2002; 39 (4): 828-33.

34. Block GA, Klassen PS, Lazarus JM, Ofsthun N, Lowrie EG, Chertow GM. Mineral metabolism, mortality, and morbidity in maintenance hemodialysis. J Am Soc Nephrol 2004; 15 (8): 2208-18.

35. Afsar A. The relationship between intact parathyroid hormone levels and daily physical activity in hemodialysis patients. Int J Artif Organs 2014; 37 (5): 350-7.

36. Marinho SM, Moraes C, Barbosa JE, Carraro Eduardo JC, Fouque D, Pelletier S, et al. Exercise Training Alters the Bone Mineral Density of Hemodialysis Patients. J Strength Cond Res 2016; 30 (10): 2918-23.

37. Junqué Jiménez A, Esteve Simó V, Tomás Bernaveu E, Paz López Ó, Iza Pinedo G, Luceño Solé I, et al. Resultado de un programa adaptado de ejercicio físico en pacientes ancianos en hemodiálisis. Enferm Nefrol. 2015; 18(1): 11-18.

38. Segura-Ortí E. Ejercicio en pacientes en hemodiálisis: revisión sistemática de la literatura. Nefrologia 2010; 30 (2): 143-269.

39. Oliveros M, Avendaño M, Bunout D, Hirsch S, de la Maza M, Pedreros C, et al. Estudio piloto sobre entrenamiento físico durante hemodiálisis. Rev Med Chile 2011; 139(8): 1046-53.
40. Cheema B, Fiatarone M. Exercise training in patients receiving maintenance hemodialysis: a systematic review of clinical trials. Am J Nephrol 2005; 25(4): 352-64.

41. Smart N, Steele M. Exercise training in haemodialysis patients: a systematic review and meta-analysis. Nephrology. 2011; 16(7): 626-32.

42. Cigarroa I, Barriga R, Michéas C, Zapata-Lamana R, Soto C, Manukian T. Efectos de un programa de ejercicio de fuerza-resistencia muscular en la capacidad funcional, fuerza y calidad de vida de adultos con enfermedad renal crónica en hemodiálisis. Rev Med Chile 2016; 144 (7): 844-52.

43. Thompson S, Tonelli M, Klarenbach S, Molzahn A. A Qualitative study to explore patient and staff perceptions of intradialytic exercise. Clin J Am Soc Nephrol 2016; 11 (6): 1024-33.

44. American Journal of Kidney Diseases. K/DOQI Clinical Practice Guidelines for Cardiovascular Disease in Dialysis Patients. [Internet]. New York: National Kidney Foundation; 2005 [Citado el 22 de marzo de 2019]. Disponible en: https:/www.kidney.org/sites/default/files/ docs/cvd_in_dialysis_composite_gl.pdf.

45. White Y, Grenyer, B. Do we encourage health or illness? A survey of exercise rehabilitation practices for patients in Australian renal units. Renal Society of Australasia Journal 2006; 2(1): 5-15. 